\title{
Partitioning 2-edge-colored Ore-type graphs by monochromatic cycles
}

\author{
János Barát \\ MTA-ELTE Geometric and Algebraic Combinatorics Research Group \\ barat@cs.elte.hu \\ and \\ Gábor N. Sárközy* \\ Alfréd Rényi Institute of Mathematics \\ Hungarian Academy of Sciences \\ Budapest, P.O. Box 127 \\ Budapest, Hungary, H-1364 \\ sarkozy.gabor@renyi.mta.hu \\ and \\ Computer Science Department \\ Worcester Polytechnic Institute \\ Worcester, MA, USA 01609 \\ gsarkozy@cs . wpi.edu
}

July 21, 2014

\begin{abstract}
Consider a graph $G$ on $n$ vertices satisfying the following Ore-type condition: for any two non-adjacent vertices $x$ and $y$ of $G$, we have $\operatorname{deg}(x)+\operatorname{deg}(y)>3 n / 2$. We conjecture that if we color the edges of $G$ with 2 colors then the vertex set of $G$ can be partitioned to two vertex disjoint monochromatic cycles of distinct colors. In this paper we prove an asymptotic version of this conjecture.
\end{abstract}

\section{Background, summary of results.}

In this paper, we consider the problem of partitioning the vertices of edge-colored graphs into monochromatic cycles. For simplicity, a colored graph means an edge-

* Research supported in part by OTKA Grant No. K104343. 
colored graph in this paper. In this context it is conventional to accept empty graphs and one-vertex graphs as a cycle (of any color) and also any edge as a cycle (in its color). With this convention one can define the cycle partition number of any colored graph $G$ as the minimum number of vertex disjoint monochromatic cycles needed to cover the vertex set of $G$. For complete graphs, [6] posed the following conjecture.

Conjecture 1. The cycle partition number of any $t$-colored complete graph $K_{n}$ is $t$.

The $t=2$ case of this conjecture was stated earlier by Lehel in a stronger form, requiring that the colors of the two cycles must be different. After some initial results [2, 8], Łuczak, Rödl and Szemerédi [19] proved Lehel's conjecture for large enough $n$, which can be considered as a birth of certain advanced applications of the Regularity Lemma. A more elementary proof, still for large enough $n$, was obtained by Allen [1]. Finally, Bessy and Thomassé [5] found a completely elementary inductive proof for every $n$.

The $t=3$ case of Conjecture 1 was solved asymptotically in [13]. Pokrovskiy [21] showed recently (with a nice elementary proof) that the path partition number of any 3 -colored $K_{n}$ is at most three (for any $n \geq 1$ ). Later Pokrovskiy [22] surprisingly found a counterexample to Conjecture 1 for all $t \geq 3$. However, in the counterexample all but one vertex can be covered by $t$ vertex disjoint monochromatic cycles, so perhaps the following weaker statement holds.

Conjecture 2. For every integer $t \geq 2$ there exists a constant $c=c(t)$ such that for any $t$-colored graph $G$ there are $t$ vertex disjoint monochromatic cycles of $G$ that cover at least $n-c$ vertices.

For general $t$, the best bound for the cycle partition number is $O(t \log t)$, see [9]. Note that it is far from obvious that the cycle partition number of $K_{n}$ can be bounded by any function of $t$.

In [3] we addressed the extension of the cycle and path partition numbers from complete graphs to arbitrary graphs $G$.

Recently, Schelp [23] suggested in a posthumous paper to strengthen certain Ramsey problems from complete graphs to graphs of given minimum degree. In particular, he conjectured that with $m=R\left(P_{n}, P_{n}\right)$, minimum degree $3 m / 4$ is sufficient to find a monochromatic path $P_{n}$ in any 2-colored graph of order $m .{ }^{1}$ Influenced by this, in [3] we posed the following

Conjecture 3. If $G$ is an n-vertex graph with $\delta(G)>3 n / 4$ then in any 2-edgecoloring of $G$, there are two vertex disjoint monochromatic cycles of different colors, which together cover $V(G)$.

\footnotetext{
${ }^{1}$ Some progress towards this conjecture have been done in [14] and [4].
} 
That is, the above mentioned Bessy-Thomassé result [5] would hold for graphs with minimum degree larger than $3 n / 4$. Note that the condition $\delta(G) \geq 3 n / 4$ is sharp (see [3]). Indeed, consider the following $n$-vertex graph, where $n=4 m$. We partition the vertex set into four parts $A_{1}, A_{2}, A_{3}, A_{4}$ with $\left|A_{i}\right|=m$. There are no edges from $A_{1}$ to $A_{2}$ and from $A_{3}$ to $A_{4}$. Edges in $\left[A_{1}, A_{3}\right],\left[A_{2}, A_{4}\right]$ are red and edges in $\left[A_{1}, A_{4}\right],\left[A_{2}, A_{3}\right]$ are blue, inside the classes any coloring is allowed. In such an edge-colored graph, there are no two vertex disjoint monochromatic cycles of different colors covering $G$, while the minimum degree is $3 m-1=3 n / 4-1$.

In [3] we proved Conjecture 3 in the following asymptotic sense.

Theorem 1. For every $\eta>0$, there is an $n_{0}(\eta)$ such that the following holds. If $G$ is an $n$-vertex graph with $n \geq n_{0}$ and $\delta(G) \geq\left(\frac{3}{4}+\eta\right) n$, then every 2-edge-coloring of $G$ admits two vertex disjoint monochromatic cycles of different colors covering at least $(1-\eta) n$ vertices of $G$.

The proof of Theorem 1 followed a method of Euczak [18]. The crucial idea of this method is that "cycles" or "paths" in a statement to be proved are replaced by "connected matchings". In a connected matching, the edges of the matching are in the same component of the graph. ${ }^{2}$ We prove first this weaker result, then we apply this to the cluster graph of a regular partition of the target graph. Through several technical details, the regularity of the partition is used to "lift back" the connected matching of the cluster graph to a path or cycle in the original graph.

In this paper we go one step further and consider graphs satisfying an Ore-type degree condition instead of a minimum degree condition. Here we call a degree condition Ore-type if it gives a lower bound on the degree sum for any two non-adjacent vertices. There has been a lot of efforts in trying to extend results from minimum degree conditions to Ore-type conditions. The first result of this type was proved by Ore [20]: If for any two non-adjacent vertices $x$ and $y$ of $G$, we have $\operatorname{deg}(x)+\operatorname{deg}(y) \geq n$, then $G$ is Hamiltonian. Some other results of this type include for example [7] (Oretype conditions for $k$-ordered Hamiltonian graphs), [15] (Ore-type results on equitable colorings) or [16] (Ore-type versions of Brooks' theorem).

Generalizing Conjecture 3 for graphs satisfying an Ore-type condition here we pose

Conjecture 4. If $G$ is an n-vertex graph such that for any two non-adjacent vertices $x$ and $y$ of $G$, we have $\operatorname{deg}(x)+\operatorname{deg}(y)>3 n / 2$, then in any 2-edge-coloring of $G$, there are two vertex disjoint monochromatic cycles of different colors, which together cover $V(G)$.

\footnotetext{
${ }^{2}$ When the edges are colored, a connected red matching is a matching in a red component.
} 
Here we prove Conjecture 4 in the following asymptotic sense.

Theorem 2. For every $\eta>0$, there is an $n_{0}(\eta)=n_{0}$ such that the following holds. If $G$ is an $n$-vertex graph with $n \geq n_{0}$ such that for any two non-adjacent vertices $x$ and $y$ of $G$, we have $\operatorname{deg}(x)+\operatorname{deg}(y) \geq\left(\frac{3}{2}+\eta\right) n$, then every 2-edge-coloring of $G$ admits two vertex disjoint monochromatic cycles of different colors covering at least $(1-\eta) n$ vertices of $G$.

The proof follows the same method as outlined above. The relaxed version of Theorem 2 for connected matchings is stated and proved in Section 2 (Theorem 3).

\section{Partitioning into connected matchings}

In this section we prove the relaxed version of our theorem for connected matchings instead of cycles.

Theorem 3. Let $G$ be an n-vertex graph, where $n$ is even and $G$ satisfies the following Ore-type condition: for any two non-adjacent vertices $x$ and $y$ of $G$, we have $\operatorname{deg}(x)+$ $\operatorname{deg}(y) \geq 3 n / 2$. If the edges of $G$ are 2 -colored with red and blue, then there exist a red connected matching and a vertex-disjoint blue connected matching, which together form a perfect matching of $G$.

Proof: Let $C_{1}$ be a largest monochromatic component, say red. Let $D=V \backslash V\left(C_{1}\right)$.

Case 1: Assume $\left|V\left(C_{1}\right)\right|<n$, i.e. $D \neq \emptyset$. Let $A$ be those vertices in $C_{1}$ that are adjacent to $D$ by a blue edge. We claim that $A \cup D$ is a connected blue component. Assume to the contrary that there is a cut in blue $\left(A_{1} \cup D_{1}, A_{2} \cup D_{2}\right)$, where $\left|A_{1} \cup D_{2}\right| \geq\left|A_{2} \cup D_{1}\right|$. Now there is no edge between $V\left(C_{1}\right) \backslash A_{2}$ and $D_{2}$. There is no red edge by the definition of $D$ and no blue edge by the assumption on the cut. Therefore if $u$ is a vertex in $V\left(C_{1}\right) \backslash A_{2}$ and $v$ is a vertex in $D_{2}$ (clearly both sets are non-empty), then $\operatorname{deg}(u)+\operatorname{deg}(v) \geq 3 n / 2$. On the other hand, $v$ is non-adjacent to all vertices of $V\left(C_{1}\right) \backslash A_{2}$ and $u$ is non-adjacent to $D_{2}$. Therefore $\operatorname{deg}(u)+\operatorname{deg}(v) \leq$ $n-1-\left|D_{2}\right|+n-1-\left|V\left(C_{1}\right) \backslash A_{2}\right| \leq 2 n-2-\left|D_{2}\right|-\left|V\left(C_{1}\right) \backslash A\right|-\left|A_{1}\right|<2 n-n / 2=3 n / 2$, a contradiction (here we used the assumption on the size of $A_{1} \cup D_{2}$ ).

Let $C_{2}$ be this blue component covering $D=V \backslash V\left(C_{1}\right)$. Let $u$ be a vertex of $C_{1} \backslash C_{2}$ and $v$ be a vertex of $C_{2} \backslash C_{1}$. Let $\left|V\left(C_{1}\right) \backslash V\left(C_{2}\right)\right|=p$ and $\left|V\left(C_{2}\right) \backslash V\left(C_{1}\right)\right|=q$, where $p \geq q>0$ by the choice of $C_{1}$. There is no edge between $u$ and $v$, in fact between $C_{1} \backslash C_{2}$ and $C_{2} \backslash C_{1}$. Therefore $\operatorname{deg}(u)+\operatorname{deg}(v) \geq 3 n / 2$. On the other hand $n-1-q \geq$ $\operatorname{deg}(u)$ and $n-1-p \geq \operatorname{deg}(v)$. It yields $2 n-2-(p+q) \geq \operatorname{deg}(u)+\operatorname{deg}(v) \geq 3 n / 2$. Therefore $p+q<n / 2$ and $\left|V\left(C_{1}\right) \cap V\left(C_{2}\right)\right|>n / 2$. 
If $\left|V\left(C_{1}\right)\right|=n$, then define $C_{2}$ as a largest blue component in $G$. Now $p=$ $\left|V\left(C_{1}\right) \backslash V\left(C_{2}\right)\right|, q=\left|V\left(C_{2}\right) \backslash V\left(C_{1}\right)\right|=0$.

Case 2: $\left|V\left(C_{1}\right)\right|=n$ and $p \leq n / 2$. Now $\left|V\left(C_{1}\right) \cap V\left(C_{2}\right)\right| \geq n / 2$, just as above.

Therefore in what follows, we unify the proof for the two cases we described so far. Let $G_{1}$ be the graph, which we obtain from $G$ by deleting the blue edges induced by $V\left(C_{1}\right) \backslash V\left(C_{2}\right)$ and the red edges induced by $V\left(C_{2}\right) \backslash V\left(C_{1}\right)$ (if these exist).

We claim there is a perfect matching in $G_{1}$. Assume the contrary. By Tutte's theorem there exists a set $X$ of vertices in $G_{1}$ such that the number of odd components in $G_{1} \backslash X$ is larger than $|X|$, which implies that $|X|<n / 2$. Let all the components of $G_{1} \backslash X$ (not just the odd ones) be $D_{1}, D_{2}, \ldots, D_{\ell}$ in increasing order of their size, $\ell \geq|X|+1$. Note that $\ell \geq 2$ always holds, even for $X=\emptyset$, as $n$ is even. Let $d_{i}=\left|V\left(D_{i}\right)\right|$ for $i=1, \ldots, \ell$ and $x=|X|$.

We claim that $\left(V\left(C_{1}\right) \cap V\left(C_{2}\right)\right) \cap\left(\cup_{i=1}^{\ell} D_{i}\right)=\emptyset$. Assume to the contrary that $u \in C_{1} \cap C_{2}$ and $u \in D_{i}$. Let $v$ be a vertex in a different $D_{j}$ (using $\ell \geq 2$ ). Clearly $u$ and $v$ are non-adjacent in $G_{1}$, but also in $G$ since we have not deleted any edge adjacent to $u$. Therefore $\operatorname{deg}_{G}(u)+\operatorname{deg}_{G}(v) \geq 3 n / 2$. Notice $\operatorname{deg}_{G_{1}}(u)=\operatorname{deg}_{G}(u)$. Now subtract the number of deleted edges adjacent to $v$, which is at most $p$ or $q$ depending on the position of $v$. We get $\operatorname{deg}_{G_{1}}(u)+\operatorname{deg}_{G_{1}}(v) \geq n$, since both $p$ and $q$ are at most $n / 2$.

On the other hand $\operatorname{deg}_{G_{1}}(u) \leq d_{i}-1+x$ and $\operatorname{deg}_{G_{1}}(v) \leq d_{j}-1+x$. Therefore $\operatorname{deg}_{G_{1}}(u)+\operatorname{deg}_{G_{1}}(v) \leq d_{i}+d_{j}+2 x-2 \leq n-1$, since $d_{i}+d_{j}+2 x-1$ is at most the number of vertices. This contradiction implies that $\left(V\left(C_{1}\right) \cap V\left(C_{2}\right)\right) \subseteq X$. However, this is impossible since $\left|V\left(C_{1}\right) \cap V\left(C_{2}\right)\right| \geq n / 2$ and $x<n / 2$. Therefore $G_{1}$ contains a perfect matching.

Case 3: $\left|V\left(C_{1}\right)\right|=n$ and $p>n / 2$, so the largest blue component has size at most $n / 2$. Again we get $G_{1}$ from $G$ by deleting the blue edges induced by $V\left(C_{1}\right) \backslash V\left(C_{2}\right)$. We claim again that there is a perfect matching in $G_{1}$ and use the same set-up as above. First we show that $V\left(C_{2}\right) \subseteq X$. As before, we select a hypothetical vertex $u$ in $C_{2} \cap D_{i}$ and a vertex $v$ in a different component $D_{j}$. Clearly $u$ and $v$ are non-adjacent in $G_{1}$, but also in $G$ since we have not deleted any edge adjacent to $u$. If there were at least $n / 2$ blue edges adjacent to $v$, then we would find a blue component larger than $C_{2}$. Therefore $\operatorname{deg}_{G}(v)-\operatorname{deg}_{G_{1}}(v)<n / 2$ and $\operatorname{deg}_{G}(u)+\operatorname{deg}_{G}(v) \geq 3 n / 2$ implies $\operatorname{deg}_{G_{1}}(u)+\operatorname{deg}_{G_{1}}(v) \geq n$. On the other hand, this is impossible since $\operatorname{deg}_{G_{1}}(u)+$ $\operatorname{deg}_{G_{1}}(v) \leq d_{i}+d_{j}+2 x-2 \leq n-1$ as in the argument above. Therefore $V\left(C_{2}\right) \subseteq X$. This implies that there is no blue component larger than $x$.

Notice that any potential edge in $G$ between two components of $G_{1} \backslash X$ is a blue edge inside $C_{1} \backslash C_{2}$ that was deleted. Let $H$ be the graph formed by the vertices in $V \backslash X$, and these crossing blue edges in $C_{1} \backslash C_{2}$. Since $x<n / 2$, we have $|V(H)|>n / 2$.

Case 3.a: Assume $x \leq n / 4$. 
We claim that $H$ is connected in the blue graph. Otherwise there exists a blue cut $(A, B)$ of $H$, where $A \cap D_{1}$ is non-empty as well as $B \cap\left(\cup_{i=2}^{\ell} D_{i}\right)$. Indeed, let us take a blue cut $(A, B)$ of $H$, where $A \cap D_{1}$ is non-empty. If $B \cap\left(\cup_{i=2}^{\ell} D_{i}\right)=\emptyset$, then $B \subseteq D_{1}$ and we reverse the roles of $A$ and $B$. Let $u$ be a vertex in $A \cap D_{1}$ and $v$ be a vertex in $B \cap D_{i}$ for some $i>1$. Now $u$ and $v$ are non-adjacent vertices in $G$. Therefore $\operatorname{deg}_{G}(u)+\operatorname{deg}_{G}(v) \geq 3 n / 2$. Since the largest blue component has size at most $x$, there are at most $x-1$ deleted blue edges at $u$ or $v$. Therefore $3 n / 2-2 x+2 \leq \operatorname{deg}_{G_{1}}(u)+\operatorname{deg}_{G_{1}}(v) \leq d_{1}-1+d_{i}-1+2 x \leq n-1$. This contradicts $x \leq n / 4$, so $H$ is indeed connected in blue. But then this is a larger blue component than $x$, a contradiction, $G_{1}$ does have a perfect matching.

Case 3.b: Assume $n / 4<x \leq n / 2$.

Here $d_{1} \leq 2$, otherwise there would be too many vertices, since $n \geq x+d_{1} \ell \geq$ $n / 4+3(n / 4+1)>n$, a contradiction.

We claim that $H$ is connected in the blue graph. This again leads to a contradiction, since we have a larger blue component than $x$. Assume the contrary and let $A$ be a blue component in $H$ that intersects $D_{1}$ and $\left.H\right|_{V(H) \backslash V(A)} \cap\left(\cup_{i=2}^{\ell} D_{i}\right)$ is non-empty. Again, if $\left(V(H) \backslash V(A) \subseteq D_{1}\right.$, then we take a blue component in $V(H) \backslash V(A)$ and that will play the role of $A$. Let $u \in A \cap D_{1}$. Let $B=\left.H\right|_{V(H) \backslash V(A)}$. Now $(A, B)$ is a cut of $H$. Let $v \in B \cap D_{i}$, where $i>1$ and $i$ is as small as possible. Now $u$ and $v$ are non-adjacent in $G$ and therefore $\operatorname{deg}_{G}(u)+\operatorname{deg}_{G}(v) \geq 3 n / 2$. On the other hand using the cut $(A, B)$, we get: $\operatorname{deg}_{G}(u) \leq n-|B|+d_{1}-1$ and $\operatorname{deg}_{G}(v) \leq n-|A|+d_{i}-1$. It implies $\operatorname{deg}_{G}(u)+\operatorname{deg}_{G}(v) \leq 2 n-(|A|+|B|)+d_{1}-1+d_{i}-1=n+x+d_{1}-1+d_{i}-1$. However this leads to a contradiction if $x+d_{1}-1+d_{i}-1<n / 2$. In what follows we prove this last inequality.

If $d_{1}=2$, then the inequality simplifies to $x+d_{i}<n / 2$. Notice that $d_{i} \leq d_{\ell}$ and $d_{\ell} \leq n-3 x$, since $\ell \geq x+1$ and $2=d_{1} \leq d_{j}$ for any $j$. Using this and $n / 4<x$ we get $x+d_{i} \leq x+d_{\ell} \leq n-2 x<n / 2$.

The other possibility is $d_{1}=1$. We have to show $x+d_{i}-1<n / 2$ or $x+d_{i} \leq n / 2$. Actually note that in the above the inequality $x+\left|D_{i} \cap A\right|<n / 2$ already leads to a contradiction, so it is sufficient to prove this. Firstly if $i=\ell$, then $A \supseteq \cup_{j=1}^{\ell-1} D_{j}$ by the choice of $i$. This is a contradiction if $|A|>x$, since now $A$ is larger than a largest blue component $C_{2}$. The only exception is $d_{j}=1$ for $1 \leq j \leq \ell-1$ and $A \cap D_{\ell}=\emptyset$. However in that case $x+\left|D_{i} \cap A\right|=x+0<n / 2$ holds. Secondly $i<\ell$. Now $\sum_{j \neq i, j \neq \ell} d_{j} \geq x-1$ and $d_{i}+d_{\ell} \geq 2 d_{i}$. If strict inequality holds in one of these, then we get the following: $n \geq x+\sum_{j \neq i, j \neq \ell} d_{j}+d_{i}+d_{\ell}>2 x-1+2 d_{i}$, which implies $n / 2 \geq x+d_{i}$ as claimed. The only case left is $i=\ell-1,1=d_{1}, \ldots, d_{i-1}$ and $d_{i}=d_{\ell}$. However this is impossible since now the number of vertices is $2 x-1+2 d_{i}$, but we started with an even $n$.

All these contradictions prove the existence of a perfect matching in $G_{1}$. Since the red and blue halves are both connected, we proved our theorem. 


\section{Applying the Regularity lemma.}

As in many applications of the Regularity Lemma, one has to handle irregular pairs, that translates to exceptional edges in the reduced graph. A graph $G$ on $n$ vertices is $\varepsilon$-perturbed if at most $\varepsilon\left(\begin{array}{l}n \\ 2\end{array}\right)$ of its edges are marked as exceptional (or perturbed). For a perturbed graph $G$, let $G^{-}$denote the graph obtained by removing all perturbed edges. First we need a perturbed version of Theorem 3. These perturbation arguments are fairly standard modifications of the original argument (see e.g. [14]). We give all details to be self-contained.

Theorem 4. For every $\eta>0$, there exist $n_{0}=n_{0}(\eta)$ and $\varepsilon_{0}=\varepsilon_{0}(\eta)(\ll \eta)$ such that the following holds. Suppose that $\varepsilon \leq \varepsilon_{0}$ and $G$ is a 2-edge-colored $\varepsilon$-perturbed graph on $n \geq n_{0}$ vertices and $G$ satisfies the following Ore-type condition: for any two non-adjacent vertices $x$ and $y$ of $G$, we have $\operatorname{deg}(x)+\operatorname{deg}(y) \geq(3 / 2+\eta) n$. All but at most $6 \sqrt{\varepsilon} n$ vertices of $G$ can be covered by the vertices of a red connected matching and a vertex-disjoint blue connected matching in $G^{-}$.

Proof: We may assume that $n$ is sufficiently large and $\varepsilon \ll \eta$. Let us start by "trimming" the graph, i.e. by deleting those vertices of $G$ that are adjacent to at least $\sqrt{\varepsilon} n$ exceptional edges. There are less than $\sqrt{\varepsilon} n$ such vertices. We may remove one more arbitrary vertex to guarantee that the number of remaining vertices is even. This way we get a slightly smaller graph $G_{\varepsilon}$ on $n^{\prime}$ vertices, where $n^{\prime}$ is even. Secondly we delete the remaining exceptional edges to form the graph $G_{\varepsilon}^{-}$. In what follows we mimic the proof of Theorem 3 replacing $G$ by $G_{\varepsilon}^{-}$.

Let $C_{1}$ be a largest monochromatic component in $G_{\varepsilon}^{-}$, say red. We have $n^{\prime}>$ $(1-\sqrt{\varepsilon}) n$. Let $D=V\left(G_{\varepsilon}^{-}\right) \backslash V\left(C_{1}\right)$.

Case 1: Assume $\left|V\left(C_{1}\right)\right|<n^{\prime}$, i.e. $D \neq \emptyset$. Let $A$ be those vertices in $C_{1}$ that are adjacent to $D$ by a blue edge. We claim that $A \cup D$ is a connected blue component. Assume to the contrary that there is a cut $\left(A_{1} \cup D_{1}, A_{2} \cup D_{2}\right)$, where $\left|A_{1} \cup D_{2}\right| \geq\left|A_{2} \cup D_{1}\right|$. Now again there is no edge in $G_{\varepsilon}^{-}$between $V\left(C_{1}\right) \backslash A_{2}$ and $D_{2}$ as before, but now there might be some exceptional edges in $G$. However if either $\left|A_{1}\right| \geq \sqrt{\varepsilon} n$ or $\left|D_{2}\right| \geq \sqrt{\varepsilon} n$, then we certainly find a pair $u, v$ that are non-adjacent in $G$ as well (so $(u, v)$ cannot be an exceptional edge) and $u \in A_{1}$ and $v \in D_{2}$. In the remaining case we have $\left|A_{2} \cup D_{1}\right| \leq\left|A_{1} \cup D_{2}\right|<2 \sqrt{\varepsilon}$. But then clearly $V\left(C_{1}\right) \backslash A_{2}>\sqrt{\varepsilon} n$ and therefore we find a non-adjacent pair $u, v$ in $G$ such that $u$ is a vertex in $V\left(C_{1}\right) \backslash A_{2}$ and $v$ is a vertex in $D_{2}$. Now for this appropriate pair of vertices $\operatorname{deg}_{G}(u)+\operatorname{deg}_{G}(v) \geq(3 / 2+\eta) n$. On the other hand, $v$ is non-adjacent to all vertices of $V\left(C_{1}\right) \backslash A_{2}$ and $u$ is non-adjacent to $D_{2}$ in the graph $G_{\varepsilon}^{-}$. Therefore $\operatorname{deg}_{G}(u)+\operatorname{deg}_{G}(v)<n-1-\left|D_{2}\right|+n-1-\left|V\left(C_{1}\right) \backslash A_{2}\right|+2 \sqrt{\varepsilon} n \leq$ $2 n-2-\left|D_{2}\right|-\left|V\left(C_{1}\right) \backslash A\right|-\left|A_{1}\right|+2 \sqrt{\varepsilon} n<2 n-n / 2+2 \sqrt{\varepsilon} n=3 n / 2+2 \sqrt{\varepsilon} n$, a contradiction using $2 \sqrt{\varepsilon} \ll \eta$ (here we used the assumption on the size of $A_{1} \cup D_{2}$ ). 
Let $C_{2}$ be this blue component of $G_{\varepsilon}^{-}$covering $V\left(G_{\varepsilon}^{-}\right) \backslash V\left(C_{1}\right)$. Let $\mid V\left(C_{1}\right) \backslash$ $V\left(C_{2}\right) \mid=p$ and $\left|V\left(C_{2}\right) \backslash V\left(C_{1}\right)\right|=q$, where $p \geq q>0$ by the choice of $C_{1}$. We claim that $p+q<(1 / 2-\eta / 2) n^{\prime}$. This clearly holds if $p, q<\sqrt{\varepsilon} n$. Otherwise $p \geq \sqrt{\varepsilon} n$ or $q \geq \sqrt{\varepsilon} n$. Therefore, we find a pair of vertices $u$ and $v$ such that $u \in V\left(C_{1}\right) \backslash V\left(C_{2}\right)$, $v \in V\left(C_{2}\right) \backslash V\left(C_{1}\right)$ and $u$ and $v$ are non-adjacent in $G$. Thus by the Ore-type condition we have $\operatorname{deg}_{G}(u)+\operatorname{deg}_{G}(v) \geq(3 / 2+\eta) n$. On the other hand $n^{\prime}-1-q \geq \operatorname{deg}_{G_{\varepsilon}^{-}}(u)$ and $n^{\prime}-1-p \geq \operatorname{deg}_{G_{\varepsilon}^{-}}(v)$. We can also use that $\operatorname{deg}_{G_{\varepsilon}^{-}}(u)+\sqrt{\varepsilon} n \geq \operatorname{deg}_{G}(u)$ and $\operatorname{deg}_{G_{\varepsilon}^{-}}(v)+\sqrt{\varepsilon} n \geq \operatorname{deg}_{G}(v)$. Now $(3 / 2+\eta) n \leq \operatorname{deg}_{G}(u)+\operatorname{deg}_{G}(v) \leq \operatorname{deg}_{G_{\varepsilon}^{-}}(u)+$ $\operatorname{deg}_{G_{\varepsilon}^{-}}(v)+2 \sqrt{\varepsilon} n \leq 2 n^{\prime}-2-(p+q)+2 \sqrt{\varepsilon} n \leq 3 n / 2+n^{\prime} / 2-2-(p+q)+2 \sqrt{\varepsilon} n$. It yields $n^{\prime} / 2+2 \sqrt{\varepsilon} n-\eta n>p+q$. Therefore $p+q<(1 / 2-\eta / 2) n^{\prime}$ since $\varepsilon \ll \eta$. This implies $\left|V\left(C_{1}\right) \cap V\left(C_{2}\right)\right|>(1 / 2+\eta / 2) n^{\prime}$.

If $\left|V\left(C_{1}\right)\right|=n^{\prime}$, then define $C_{2}$ as a largest blue component in $G_{\varepsilon}^{-}$. Now $p=$ $\left|V\left(C_{1}\right) \backslash V\left(C_{2}\right)\right|, q=\left|V\left(C_{2}\right) \backslash V\left(C_{1}\right)\right|=0$.

Case 2: $\left|V\left(C_{1}\right)\right|=n^{\prime}$ and $p \leq(1 / 2-\eta / 2) n^{\prime}$. Then again $\left|V\left(C_{1}\right) \cap V\left(C_{2}\right)\right| \geq$ $(1 / 2+\eta / 2) n^{\prime}$, just as above.

Therefore in what follows, we unify the proof for the two cases we described so far. Let $G_{1}$ be the graph, which we obtain from $G_{\varepsilon}^{-}$by deleting the blue edges induced by $V\left(C_{1}\right) \backslash V\left(C_{2}\right)$ and the red edges induced by $V\left(C_{2}\right) \backslash V\left(C_{1}\right)$ (if these exist).

We claim there is a perfect matching in $G_{1}$. Assume the contrary. By Tutte's theorem there exists a set $X$ of vertices in $G_{1}$ such that the number of odd components in $G_{1} \backslash X$ is larger than $|X|$, which implies that $|X|<n / 2$. Let all the components of $G_{1} \backslash X$ (not just the odd ones) be $D_{1}, D_{2}, \ldots, D_{\ell}$ in increasing order of their size, $\ell \geq|X|+1$. Note that $\ell \geq 2$ always holds, even for $X=\emptyset$, as $n^{\prime}$ is even. Let $d_{i}=\left|V\left(D_{i}\right)\right|$ for $i=1, \ldots, \ell$ and $x=|X|$.

We claim $\left|\left(V\left(C_{1}\right) \cap V\left(C_{2}\right)\right) \cap \cup_{i=1}^{\ell} D_{i}\right| \leq 2 \sqrt{\varepsilon} n$. Assume the contrary. Now we want to copy the corresponding part of the proof of Theorem 3. Although some nonadjacent $u, v$ pairs in $G_{1}$ might be connected by an exceptional edge in $G$, the size of $\left|\left(V\left(C_{1}\right) \cap V\left(C_{2}\right)\right) \cap \cup_{i=1}^{\ell} D_{i}\right|$ now assures that we find a non-adjacent pair as follows. We can find an index $j$ such that $D_{j} \cap\left(V\left(C_{1}\right) \cap V\left(C_{2}\right)\right)=U$ is non-empty. We can think of $U$ as a collection of potential $u$ 's. Let $\bar{D}_{j}=\cup_{i=1, i \neq j}^{\ell} D_{i}$. If $\left|\bar{D}_{j}\right| \geq \sqrt{\varepsilon} n$, then pick any vertex $u \in U$. There are less than $\sqrt{\varepsilon} n$ exceptional edges adjacent to $u$. Therefore, we find a vertex $v \in \bar{D}_{j}$ that is non-adjacent to $u$ in $G$. If $\left|\bar{D}_{j}\right|<\sqrt{\varepsilon} n$, then $|U| \geq \sqrt{\varepsilon} n$. Now pick a vertex $v \in \bar{D}_{j}$ (using $\ell \geq 2$ ). There are less than $\sqrt{\varepsilon} n$ exceptional edges adjacent to $v$. Therefore, we find a vertex $u \in U$ that is non-adjacent to $v$ in $G$.

Now we may use the Ore-type condition $\operatorname{deg}_{G}(u)+\operatorname{deg}_{G}(v) \geq(3 / 2+\eta) n$. Notice $\operatorname{deg}_{G_{1}}(u) \geq \operatorname{deg}_{G}(u)-\sqrt{\varepsilon} n$. Let us subtract the number of deleted non-exceptional edges adjacent to $v$, which is at most $p$ or $q$ depending on the position of $v$. We get $\operatorname{deg}_{G_{1}}(u)+\operatorname{deg}_{G_{1}}(v) \geq \operatorname{deg}_{G}(u)-\sqrt{\varepsilon} n+\operatorname{deg}_{G}(v)-p-\sqrt{\varepsilon} n \geq(3 / 2+\eta) n-$ $2 \sqrt{\varepsilon} n-p \geq n$, since both $p$ and $q$ are less than $n / 2$ and $\varepsilon \ll \eta$. On the other hand 
$\operatorname{deg}_{G_{1}}(u) \leq d_{i}-1+x$ and $\operatorname{deg}_{G_{1}}(v) \leq d_{j}-1+x$. Therefore $\operatorname{deg}_{G_{1}}(u)+\operatorname{deg}_{G_{1}}(v) \leq$ $d_{i}+d_{j}+2 x-1-1 \leq n^{\prime}-1 \leq n-1$, since $d_{i}+d_{j}+2 x-1$ is at most the number of vertices in $G_{1}$. This contradiction implies $\left|\left(V\left(C_{1}\right) \cap V\left(C_{2}\right)\right) \cap \cup_{i=1}^{\ell} D_{i}\right| \leq 2 \sqrt{\varepsilon}$. Using this we get $n / 2+2 \sqrt{\varepsilon} n>x+2 \sqrt{\varepsilon} n \geq\left|V\left(C_{1}\right) \cap V\left(C_{2}\right)\right| \geq(1 / 2+\eta / 2) n^{\prime}>$ $(1 / 2+\eta / 2)(1-\sqrt{\varepsilon}) n$. However, this is a contradiction since $\varepsilon \ll \eta$. Therefore $G_{1}$ contains a perfect matching.

Case 3: $\left|V\left(C_{1}\right)\right|=n^{\prime}$ and $p>(1 / 2-\eta / 2) n^{\prime}$, so the largest blue component of $G_{\varepsilon}^{-}$ has size at most $(1 / 2+\eta / 2) n^{\prime}$. We claim in this case that there is a matching in $G_{1}$ covering all but at most $5 \sqrt{\varepsilon} n$ vertices of $G_{1}$. We use the same set-up and notation as previously. Thus by Tutte's theorem now we have slightly more components than before: $\ell \geq|X|+5 \sqrt{\varepsilon} n$. This implies $x=|X|<n^{\prime} / 2-2 \sqrt{\varepsilon} n$.

We can show $\left|V\left(C_{2}\right) \cap \cup_{i=1}^{\ell} D_{i}\right| \leq 2 \sqrt{\varepsilon} n$ as before. Assume the contrary and as above select a vertex $u$ in $C_{2} \cap D_{i}$ and a vertex $v$ in a different component $D_{j}$. Clearly $u$ and $v$ are non-adjacent in $G_{1}$, but also in $G$ since we have not deleted any edge adjacent to $u$. If there were at least $(1 / 2+\eta / 2) n\left(\geq(1 / 2+\eta / 2) n^{\prime}\right)$ deleted nonexceptional blue edges adjacent to $v$, then we would find a blue component larger than $C_{2}$. Therefore $\operatorname{deg}_{G}(v)-\operatorname{deg}_{G_{1}}(v)<(1 / 2+\eta / 2) n+\sqrt{\varepsilon} n, \operatorname{deg}_{G_{1}}(u) \geq \operatorname{deg}_{G}(u)-\sqrt{\varepsilon} n$ and $\operatorname{deg}_{G}(u)+\operatorname{deg}_{G}(v) \geq(3 / 2+\eta) n$ imply $\operatorname{deg}_{G_{1}}(u)+\operatorname{deg}_{G_{1}}(v) \geq(1+\eta / 2) n-2 \sqrt{\varepsilon} n>$ $n$. On the other hand, this is impossible since $\operatorname{deg}_{G_{1}}(u)+\operatorname{deg}_{G_{1}}(v) \leq d_{i}+d_{j}+2 x-$ $1-1 \leq n^{\prime}-1<n$ as in the previous argument. Therefore $\left|V\left(C_{2}\right) \cap \cup_{i=1}^{\ell} D_{i}\right| \leq 2 \sqrt{\varepsilon} n$. This implies that there is no blue component larger than $x+2 \sqrt{\varepsilon} n$.

Notice that any non-exceptional edge in $G$ between two components of $G_{1} \backslash X$ is a blue edge inside $C_{1} \backslash C_{2}$ that was deleted. Let $H$ be the graph formed by the vertices in $V\left(G_{1}\right) \backslash X$, and these crossing non-exceptional blue edges in $C_{1} \backslash C_{2}$. Now we have $x=|X|<n^{\prime} / 2-2 \sqrt{\varepsilon} n$ and therefore $|V(H)|>n^{\prime} / 2+2 \sqrt{\varepsilon} n$.

Case 3.a: Assume $x \leq(1+\eta) n / 4$.

We claim that $H$ is connected in the blue graph except for possibly $2 \sqrt{\varepsilon} n$ vertices. This leads to the final contradiction, since $|V(H)|-2 \sqrt{\varepsilon} n>n^{\prime} / 2>x+2 \sqrt{\varepsilon} n$. (We found a blue connected component larger than the size of a largest.)

Assume the contrary. Then there exists a blue cut $(A, B)$ of $H$ where we have $|A|,|B|>2 \sqrt{\varepsilon} n, A \cap D_{1}$ is non-empty and $\left|B \cap \cup_{i=2}^{\ell} D_{i}\right| \geq \sqrt{\varepsilon} n$. Indeed, let us take a blue cut $(A, B)$ of $H$, where $|A|,|B|>2 \sqrt{\varepsilon} n$ and $A \cap D_{1}$ is non-empty. If $\left|B \cap \cup_{i=2}^{\ell} D_{i}\right|<\sqrt{\varepsilon} n$, then $\left|B \cap D_{1}\right| \geq \sqrt{\varepsilon} n$ and we reverse the roles of $A$ and $B$. Let $u$ be a vertex in $A \cap D_{1}$. Since $\left|B \cap \cup_{i=2}^{\ell} D_{i}\right| \geq \sqrt{\varepsilon} n$, we find a vertex $v$ in $B \cap \bar{D}_{1}$ such that $u$ and $v$ are non-adjacent in $G$. Therefore $\operatorname{deg}_{G}(u)+\operatorname{deg}_{G}(v) \geq$ $(3 / 2+\eta) n$. Since the largest blue component has size at most $x+2 \sqrt{\varepsilon} n$, there are at most $x+2 \sqrt{\varepsilon} n-1$ deleted non-exceptional blue edges at $u$ or $v$. Therefore $(3 / 2+\eta) n-2 x-4 \sqrt{\varepsilon} n+2-2 \sqrt{\varepsilon} n \leq \operatorname{deg}_{G_{1}}(u)+\operatorname{deg}_{G_{1}}(v) \leq d_{1}-1+d_{i}-1+2 x \leq n-1$. This yields $n / 2+\eta n-6 \sqrt{\varepsilon} n \leq 2 x$. This contradicts $x \leq(1+\eta) n / 4$, since $\varepsilon \ll \eta$.

Case 3.b: Assume $(1+\eta) n / 4<x<n^{\prime} / 2-2 \sqrt{\varepsilon} n$. 
Here $d_{1} \leq 2$, otherwise there would be too many vertices, since $n \geq x+d_{1} \ell>$ $x+3 x=4 x>(1+\eta) n$, a contradiction.

We claim again that $H$ is connected in the blue graph except for possibly $2 \sqrt{\varepsilon} n$ vertices, a contradiction again, since $|V(H)|-2 \sqrt{\varepsilon} n>n^{\prime} / 2>x+2 \sqrt{\varepsilon} n$. (We found a blue connected component larger than the size of a largest.) Assume the contrary and let $A$ be a blue component in $H$ that intersects $D_{1}$. Let $u \in A \cap D_{1}$. Let $B=\left.H\right|_{V(H) \backslash V(A)}$. Now $(A, B)$ is a cut of $H$. We may assume $|B| \geq 2 \sqrt{\varepsilon} n$ (since otherwise we are done) and thus $\left|B \cap\left(\cup_{i=2}^{\ell} D_{i}\right)\right| \geq \sqrt{\varepsilon} n$ (using $d_{1} \leq 2$ ). Let $v \in B \cap D_{i}$ such that $u$ and $v$ are non-adjacent in $G$ and $i>1$ is as small as possible. Now $u$ and $v$ are non-adjacent in $G$ and therefore $\operatorname{deg}_{G}(u)+\operatorname{deg}_{G}(v) \geq(3 / 2+\eta) n$. On the other hand using the cut $(A, B)$, we get: $\operatorname{deg}_{G}(u) \leq n-|B|+d_{1}-1+\sqrt{\varepsilon} n$ and $\operatorname{deg}_{G}(v) \leq n-|A|+d_{i}-1+\sqrt{\varepsilon} n$. It implies $(3 / 2+\eta) n \leq \operatorname{deg}_{G}(u)+\operatorname{deg}_{G}(v) \leq 2 n-(|A|+$ $|B|)+d_{1}-1+d_{i}-1+2 \sqrt{\varepsilon} n=2 n-n^{\prime}+x+d_{1}-1+d_{i}-1+2 \sqrt{\varepsilon} n=n+x+d_{1}+d_{i}-2+3 \sqrt{\varepsilon} n$. However this is a contradiction if $x+d_{1}+d_{i}-2+3 \sqrt{\varepsilon} n<n / 2+\eta n$. Using $d_{1} \leq 2$, it suffices to prove $x+d_{i}+3 \sqrt{\varepsilon} n<n / 2+\eta n$, or $x+d_{i}<(1+\eta) n / 2$. In what follows we prove this last inequality.

Let $d_{1}=2$. Notice that $d_{i} \leq d_{\ell}$ and $d_{\ell} \leq n-3 x$, since $\ell>x$ and $2=d_{1} \leq d_{j}$ for any $j$. Using this and $(1+\eta) n / 4<x$ we get $x+d_{i} \leq x+d_{\ell} \leq n-2 x<n-(1+\eta) n / 2<$ $n / 2<(1+\eta) n / 2$, as desired.

The other possibility is $d_{1}=1$. Firstly if $i=\ell$, then $A \supseteq \cup_{j=1}^{\ell-1} D_{j}$. This is a contradiction since $|A|>x+2 \sqrt{\varepsilon} n$, we have a blue component that is larger than a largest blue component $C_{2}$. Secondly $i<\ell$. Now $\sum_{j \neq i, j \neq \ell} d_{j} \geq x$ and $d_{i}+d_{\ell} \geq 2 d_{i}$. Thus we get the following: $n \geq x+\sum_{j \neq i, j \neq \ell} d_{j}+d_{i}+d_{\ell}>2 x+2 d_{i}$, which implies $x+d_{i} \leq n / 2<(1+\eta) n / 2$, as claimed.

All these contradictions prove the existence of a matching in $G_{1}$ of the desired size (covering all but at most $\sqrt{\varepsilon} n+5 \sqrt{\varepsilon} n=6 \sqrt{\varepsilon} n$ vertices of $G$ ). Since the red and blue halves are both connected, we proved our theorem.

\section{Building cycles from connected matchings.}

Next we show how to prove Theorem 2 from Theorem 4 and the Szemerédi Regularity Lemma [24]. The material of this section is fairly standard by now (see [3, 9, 10, 11, $12,13]$ so we omit some of the details.

We need a 2-edge-colored version of the Szemerédi Regularity Lemma. ${ }^{3}$

Lemma 1. For every integer $m_{0}$ and positive $\varepsilon$, there is an $M_{0}=M_{0}\left(\varepsilon, m_{0}\right)$ such that for $n \geq M_{0}$ the following holds. For any $n$-vertex graph $G$, where $G=G_{1} \cup G_{2}$

\footnotetext{
${ }^{3}$ For background, this variant and other variants of the Regularity Lemma see [17].
} 
with $V\left(G_{1}\right)=V\left(G_{2}\right)=V$, there is a partition of $V$ into $\ell+1$ clusters $V_{0}, V_{1}, \ldots, V_{\ell}$ such that

- $m_{0} \leq \ell \leq M_{0},\left|V_{1}\right|=\left|V_{2}\right|=\cdots=\left|V_{\ell}\right|=L,\left|V_{0}\right|<\varepsilon n$,

- apart from at most $\varepsilon\left(\begin{array}{l}\ell \\ 2\end{array}\right)$ exceptional pairs, all pairs $\left.G_{s}\right|_{V_{i} \times V_{j}}$ are $\varepsilon$-regular, where $1 \leq i<j \leq \ell$ and $1 \leq s \leq 2$.

Proof: Let $\varepsilon \ll \rho \ll \eta \ll 1, m_{0}$ sufficiently large compared to $1 / \varepsilon$ and $M_{0}$ obtained from Lemma 1 . Let $G$ be a graph on $n>M_{0}$ vertices such that for any two non-adjacent vertices $x$ and $y$ of $G$, we have $\operatorname{deg}(x)+\operatorname{deg}(y) \geq\left(\frac{3}{2}+\eta\right) n$. Consider a 2-edge-coloring of $G$, that is $G=G_{1} \cup G_{2}$. We apply Lemma 1 to $G$. We obtain a partition of $V$, that is $V=\cup_{0 \leq i \leq \ell} V_{i}$.

Define the following reduced graph $G^{R}$ : The vertices $p_{1}, \ldots, p_{\ell}$ of $G^{R}$ correspond to the clusters, and there is an edge between vertices $p_{i}$ and $p_{j}$ if the pair $\left(V_{i}, V_{j}\right)$ is either exceptional $^{4}$, or if it is $\varepsilon$-regular in both $G_{1}$ and $G_{2}$ with density in $G$ exceeding $\rho$. Thus note that $G^{R}$ is an $\varepsilon$-perturbed graph where a non-edge is a regular pair where the density is at most $\rho$. The edge $p_{i} p_{j}$ is colored by the color, which is used on most edges from $G\left[V_{i}, V_{j}\right]$ (the bipartite subgraph of $G$ with edges between $V_{i}$ and $V_{j}$ ). If the pair is non-exceptional, then the density of this majority color is still at least $\rho / 2$ in $G\left[V_{i}, V_{j}\right]$. This defines a 2-edge-coloring $G^{R}=G_{1}^{R} \cup G_{2}^{R}$.

We claim that $G^{R}$ satisfies a similar Ore-type condition: for any two non-adjacent vertices $p_{i}$ and $p_{j}$ of $G^{R}$, we have $\operatorname{deg}_{G^{R}}\left(p_{i}\right)+\operatorname{deg}_{G^{R}}\left(p_{j}\right) \geq\left(\frac{3}{2}+\frac{\eta}{2}\right) \ell$. Indeed, let $p_{i}$ and $p_{j}$ be non-adjacent in $G^{R}$ and consider the corresponding clusters $V_{i}$ and $V_{j}$. By definition the number of non-edges in $G\left[V_{i}, V_{j}\right]$ is at least $(1-\rho)\left|V_{i}\right|\left|V_{j}\right|=(1-\rho) L^{2}$. For each of these non-edges we can use the Ore-condition in $G$ so we get the following estimate

$$
\sum_{u \in V_{i}} \sum_{v \in V_{j}}\left(\operatorname{deg}_{G}(u)+\operatorname{deg}_{G}(v)\right) \geq(1-\rho) L^{2}\left(\frac{3}{2}+\eta\right) n .
$$

On the other hand we can get the following upper bound for this quantity

$$
\sum_{u \in V_{i}} \sum_{v \in V_{j}}\left(\operatorname{deg}_{G}(u)+\operatorname{deg}_{G}(v)\right) \leq L^{3}\left(\operatorname{deg}_{G^{R}}\left(p_{i}\right)+\operatorname{deg}_{G^{R}}\left(p_{j}\right)\right)+2 \varepsilon n L^{2}+2 \rho n L^{2},
$$

where the last 2 error terms come from the edges to $V_{0}$, and from the regular pairs with density at most $\rho$. However, from this we get

$$
\operatorname{deg}_{G^{R}}\left(p_{i}\right)+\operatorname{deg}_{G^{R}}\left(p_{j}\right)>\left(\frac{3}{2}+\frac{\eta}{2}\right) \frac{n}{L} \geq\left(\frac{3}{2}+\frac{\eta}{2}\right) \ell,
$$

as desired.

\footnotetext{
${ }^{4}$ That is, $\varepsilon$-irregular in $G_{1}$ or in $G_{2}$. Also, these edges are marked exceptional in $G^{R}$.
} 
Applying Theorem 4 to the 2-colored and $\varepsilon$-perturbed $G^{R}$, we get a connected matching in $\left(G_{1}^{R}\right)^{-}$and a vertex-disjoint connected matching in $\left(G_{2}^{R}\right)^{-}$, which together cover most of $G^{R}$. Finally, we lift the connected matchings back to cycles in the original graph using the following ${ }^{5}$ lemma in our context.

Lemma 2. Assume that there is a monochromatic connected matching $M$ (say in $\left.\left(G_{1}^{R}\right)^{-}\right)$saturating at least $c\left|V\left(G^{R}\right)\right|$ vertices of $G^{R}$, for some positive constant $c$. Then in the original $G$ there is a monochromatic cycle in $G_{1}$ covering at least $c(1-3 \varepsilon) n$ vertices.

This completes the proof. Indeed, the number of vertices left uncovered in $G$ is at most $f(\varepsilon) n \leq \eta n$, using our choice of $\varepsilon$. Here the uncovered parts come from Theorem 4, Lemma 2 and $V_{0}$.

\section{References}

[1] P. Allen, Covering two-edge-coloured complete graphs with two disjoint monochromatic cycles, Combinatorics, Probability and Computing, 17 (2008), pp. 471-486.

[2] J. Ayel, Sur l'existence de deux cycles supplementaires unicolorés, disjoints et de couleurs differentes dans un graphe complet bicolore, Thesis, University of Grenoble, (1979).

[3] J. Balogh, J. Barát, D. Gerbner, A. Gyárfás, G. Sárközy, Partitioning 2-edgecolored graphs by monochromatic paths and cycles. Combinatorica to appear.

[4] F.S. Benevides, T. Łuczak, A. Scott, J. Skokan, M. White, Monochromatic cycles and the monochromatic circumference in 2-coloured graphs, Combin. Probab. Comput. 21 (2012), pp. 57-87.

[5] S. Bessy, S. Thomassé, Partitioning a graph into a cycle and an anticycle, a proof of Lehel's conjecture, Journal of Combinatorial Theory, Series B 100 (2010), pp. 176-180.

[6] P. Erdős, A. Gyárfás, L. Pyber, Vertex coverings by monochromatic cycles and trees, Journal of Combinatorial Theory, Series B 51 (1991), pp. 90-95.

[7] R. J. Faudree, R. J. Gould, A. V. Kostochka, L. Lesniak, I. Schiermeyer, A. Saito, Degree conditions for k -ordered Hamiltonian graphs, Journal of Graph Theory 42 (2003), pp. 199-210.

\footnotetext{
${ }^{5}$ As in $[10,11,12,13]$.
} 
[8] A. Gyárfás, Vertex coverings by monochromatic paths and cycles, Journal of Graph Theory 7 (1983), pp. 131-135.

[9] A. Gyárfás, M. Ruszinkó, G. Sárközy, E. Szemerédi, An improved bound for the monochromatic cycle partition number, Journal of Combinatorial Theory, Series $B 96$ (2006), pp. 855-873.

[10] A. Gyárfás, J. Lehel, G. N. Sárközy, R. H. Schelp, Monochromatic Hamiltonian Berge cycles in colored complete hypergraphs, Journal of Combinatorial Theory, Ser. B 98 (2008), pp. 342-358.

[11] A. Gyárfás, M. Ruszinkó, G. N. Sárközy, E. Szemerédi, Tripartite Ramsey numbers for paths, Journal of Graph Theory 55 (2007), pp. 164-170.

[12] A. Gyárfás, M. Ruszinkó, G. Sárközy, E. Szemerédi, Three-color Ramsey numbers for paths, Combinatorica 27 (2007), pp. 35-69.

[13] A. Gyárfás, M. Ruszinkó, G. Sárközy, E. Szemerédi, Partitioning 3-colored complete graphs into three monochromatic cycles, Electronic Journal of Combinatorics 18 (2011), Nr. 53.

[14] A. Gyárfás, G. N. Sárközy, Star versus two stripes Ramsey numbers and a conjecture of Schelp, Combin. Probab. Comput. 21 (2012), pp. 179-186.

[15] H. A. Kierstead, A. V. Kostochka, An Ore-type theorem on equitable coloring, Journal of Combinatorial Theory, Ser. B 98 (2008), pp. 226-234.

[16] H. A. Kierstead, A. V. Kostochka, Ore-type versions of Brooks' theorem, Journal of Combinatorial Theory, Ser. B 99 (2009), pp. 298-305.

[17] J. Komlós and M. Simonovits, Szemerédi's Regularity Lemma and its applications in Graph Theory, in Combinatorics, Paul Erdős is Eighty (D. Miklós, V.T. Sós and T. Szőnyi, Eds.), pp. 295-352, Bolyai Society Mathematical Studies, Vol. 2, Budapest, 1996.

[18] T. Euczak, $R\left(C_{n}, C_{n}, C_{n}\right) \leq(4+o(1)) n$, Journal of Combinatorial Theory, Ser. $B 75$ (1999), pp. 174-187.

[19] T. Łuczak, V. Rödl, E. Szemerédi, Partitioning two-colored complete graphs into two monochromatic cycles, Probability, Combinatorics and Computing 7 (1998), pp. 423-436.

[20] O. Ore, Note on Hamilton circuits. Amer. Math. Monthly 67 (1960) 55. 
[21] A. Pokrovskiy, Partitioning 3-coloured complete graphs into three monochromatic paths, Electronic Notes in Discrete Mathematics 38 (2011), pp. 717-722.

[22] A. Pokrovskiy, Partitioning edge-coloured complete graphs into monochromatic cycles and paths, Journal of Combinatorial Theory, Ser. B, 106 (2014), pp. 70-97

[23] R. H. Schelp, A minimum degree condition on a Ramsey graph which arrows a path, submitted to Discrete Mathematics.

[24] E. Szemerédi, Regular partitions of graphs, Colloques Internationaux C.N.R.S. № 260 - Problèmes Combinatoires et Théorie des Graphes, Orsay (1976), pp. 399-401. 